\section{Biases in cold fusion data}

SIR-Jones et al. ${ }^{1}$ have claimed to have observed 'cold' nuclear fusion. Their data, however, have a peculiar characteristic, which may indicate a systematic bias in the data collection procedure.

In Fig. 4 of ref. 1, which gives the foreground-to-background ratio from 14 different experimental runs, each of the data points indicates a positive excess,
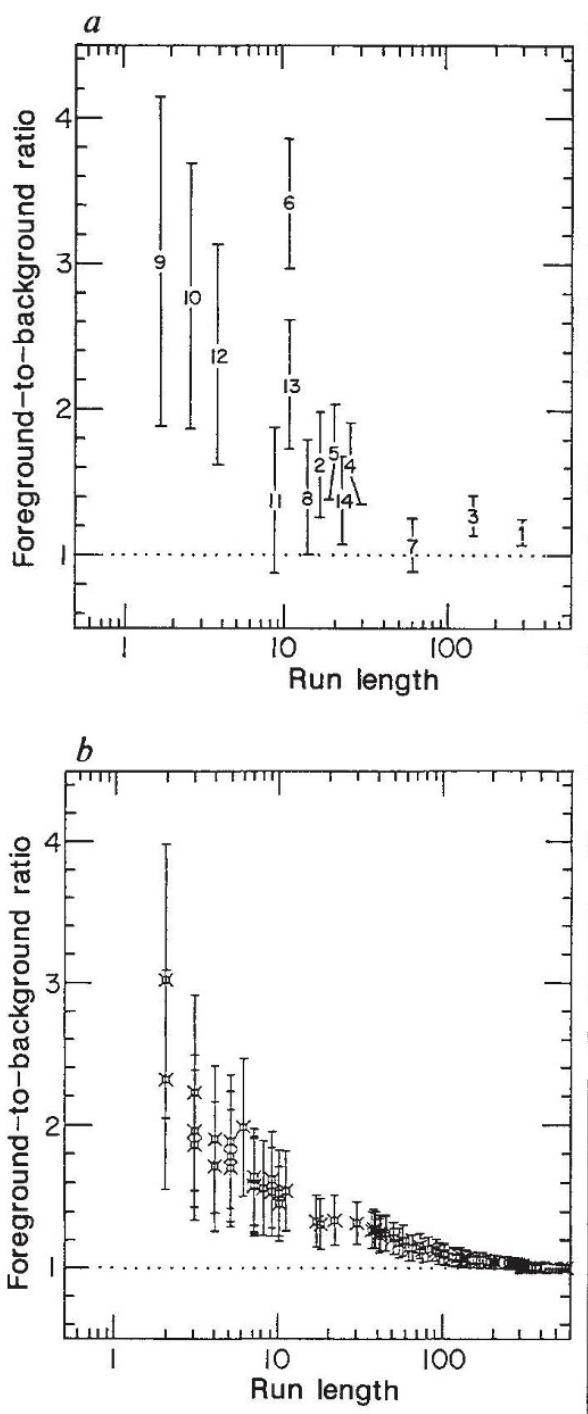

a, Results from Jones et al. ${ }^{1}$ plotted against run length. Run length is scaled by the square of the stated errors. Data points are labelled by run number. $b$, Results from a simulation of a biased-run-length selection algorithm. Only statistical errors are shown.

which is taken as evidence for cold fusion. Runs with largest signal also have the largest statistical errors, indicating that for these runs data were collected for only a short time. Our figure $a$ shows the data of Jones et al. for 14 runs replotted as a function of the run length. (Here we have determined the run length by assuming that the stated error is statistical.) It is evident that the data collection time varied enormously from run to run. Although the apparent systematic correla- tion of signal rate and run length may be explained by Jones' hypothesis of a timevarying signal, there are other causes that do not require the existence of a true signal at all.

If the length of the run is chosen on the basis of information obtained from the data collected, the experimenter can exert a surprisingly strong influence on the final result. In the extreme case, the experimenter systematically stops runs when the difference between the foreground and background happens to be positive. Statistical or random systematic variations can produce this difference even when there is no real signal. Such a bias would produce a strong correlation between the deviation from the null result and the size of the statistical error. We have modelled several different scenarios for experiments affected by this bias.

The results of one of our simulations are shown in $b$ of the figure. The number of events assigned to foreground and background are chosen randomly at each time step from identical guassian distributions. The mean value of the distribution changes at each step according to a guassian distribution with a mean of 2.2 counts per step and a standard deviation of 1.9 counts per step (this models a large random systematic variation). Values of less than zero are treated as zero. The signal (foreground minus background counts) is evaluated at each step using all the data collected for the run. The run is ended and labelled as 'successful' if any of the following conditions are met: (1) the total signal exceeds 20 counts; (2) the total signal exceeds $(4+0.5$ $x$ number of steps) counts - this ensures that there are some short runs; (3) the signal exceeds 4 counts, and the run has lasted for more than 300 steps. Runs exceeding 600 steps without meeting any of the above criteria are terminated and labelled as 'failures'.

About 75 per cent of the runs are 'successful' despite the absence of a 'true' signal. For 1,000 simulated runs, there are 15 groups of 10 or more successful runs in a row. The results for the first 100 successful runs are shown $b$ of the figure. The exact fraction and distribution of runs that exhibit positive signals depends on the algorithm used to terminate the run. Nevertheless, the general correlation between signal strength and error (or run length) is evident and reproducible for each of the six different algorithms that we have considered.

We find that statistical fluctuations alone cannot quantitatively reproduce the high foreground-to-background ratios reported by Jones et al. ${ }^{1}$; but systematic fluctuations to realistic size (such as those introduced in the above scenario) may be sufficient to reproduce them. For exam- ple, a small time-dependent gain shift in the neutron detector used by Jones et al., combined with a selection bias, can lead to an apparently positive result. The shape of the observed neutron energy spectrum and the location of the expected signal peak makes gain stability particularly important.

Except for run 6, which shows the most significant variation from the null hypothesis, the results of ref. 1 do seem to follow the trend predicted for a run-length selection bias. It is impossible to prove that selection bias is the origin of the 'positive' result obtained in ref. 1 , but we emphasize that it is a potentially dangerous feature of experiments in which the running time is not selected a priori. The presence of the characteristic correlation makes it difficult to evaluate the statistical significance of the conclusion of Jones et $a l$. in the absence of detailed information about the criteria used to select the run times.

STUART FREEDMAN DANIEL KRAKAUER

Argonne National Laboratory,

Argonne,

IIlinois 60439,

USA

JONES ET AL. REPLY-Freedman and Krakauer correctly state that by judiciously stopping a random Poisson process the experimenter can bias the count data to give an apparent statistically significant effect. The key to creating such a bias from a noise-only signal is that the decision to stop the experiment be based on the data of that run observed to date; when the decision to stop is independent of the data collected, the bias cannot be created. In our case, the decision to terminate a run (or run sequence as in the case of runs 5-7) was independent of the data collected previously during that run, and we undertook procedures to guard against systematic errors.

The first four of our foreground and corresponding background runs were of arbitrary length, determined by convenience in changing electrodes and electrolytes. The data were collected on a multichannel analyser, without any review of the time histories of data acquisition. We did notice in the combined foreground runs the appearance of a small signal at approximately the correct energy for fusion neutrons (2.5 MeV). After run 5 had been running for $2-3$ hours we noticed that the rate increased dramatically. We stopped run 5 and immediately startedrun 6 in order to isolate this set of data for separate analysis. When the rate decreased several hours later, we started run 7 . This run was terminated at an arbitrary time, so that runs 5 to 7 had a total length that was independent of the data collected. (For run 6 we checked that emission rates of neutrons with energy 